\title{
Long-Term Intraocular Pressure-Lowering Effects and Adverse Events of Ripasudil in Patients with Glaucoma or Ocular Hypertension over 24 Months
}

Hidenobu Tanihara $\cdot$ Takahiko Kakuda $\cdot$ Tetsuro Sano •

Takashi Kanno $\cdot$ Yuji Kurihara

Received: October 28, 2021 / Accepted: December 15, 2021 / Published online: February 12, 2022

(C) The Author(s) 2022

\section{ABSTRACT}

Introduction: Glaucoma is a leading cause of irreversible blindness and ripasudil was the first Rho kinase inhibitor approved as antiglaucoma medication. Here we present the final analysis of the ROCK-J study, a large-scale post-marketing surveillance study to evaluate the long-term safety and effectiveness of ripasudil in Japanese patients with glaucoma or ocular hypertension in a real-word clinical setting.

Methods: ROCK-J was a 24-month, prospective, open-label, observational study that included ripasudil-naïve patients with glaucoma or ocular hypertension who were initiating treatment with ripasudil according to the Japanese approved indication between June 1, 2015 and April 30, 2017. The primary safety endpoint was

Supplementary Information The online version contains supplementary material available at https:// doi.org/10.1007/s12325-021-02023-y.

\section{H. Tanihara}

Kumamoto University Hospital, 1-1-1 Honjo, Chuoku, Kumamoto 860-8556, Japan

H. Tanihara $(\bowtie)$

Biei Municipal Hospital, 3-8-35, Naka-machi, Biei Town, Kamikawa-gun, Hokkaido 071-0207, Japan e-mail: tanihara@pearl.ocn.ne.jp

T. Kakuda $\cdot$ T. Sano $\cdot$ T. Kanno $\cdot$ Y. Kurihara Post Marketing Surveillance Department, Kowa Co., Ltd., 4-14, Nihonbashi-honcho 3-chome, Chuo-ku, Tokyo 103-8433, Japan the incidence of adverse drug reactions (ADRs) (including blepharitis, plus assessment of its background factors); the primary efficacy endpoint was change in intraocular pressure (IOP) from baseline to 24 months.

Results: A total of 3374 Japanese patients with glaucoma or ocular hypertension were evaluated for safety and 3178 for effectiveness of ripasudil over a mean 524.5-day observational period. Overall, 853 (25.3\%) patients experienced adverse drug reactions; the most common were blepharitis (8.6\%), conjunctival hyperemia (8.5\%), and conjunctivitis (6.3\%). Multivariate analyses demonstrated that patients were more likely to experience the ADR blepharitis with ripasudil treatment if they were female (hazard ratio [HR] 1.307; $p=0.040$ ), had comorbid or a previous history of blepharitis (HR 2.178; $p=0.001$ ), or had a history of allergy to pollen (HR $1.645 ; p=0.003$ ) or medication (HR 2.276; $p<0.001)$. IOP decreased significantly from baseline with ripasudil; the leastsquares mean \pm standard error change in IOP from baseline to 24 months was $-2.6 \pm 0.1 \mathrm{mmHg}(p<0.001)$. Significant IOP changes were seen in four types of glaucoma, namely primary open-angle glaucoma, normaltension glaucoma, primary angle-closure glaucoma, and secondary glaucoma, and ocular hypertension.

Conclusion: Ripasudil was safe and effective as an antiglaucoma medication with no new safety signals identified and significant reductions in 
IOP maintained over 24 months of treatment.

Keywords: Blepharitis;

Effectiveness;

Glaucoma; Intraocular pressure; Observational study; Post-marketing surveillance; Ripasudil; ROCK inhibitor; Safety

\section{Key Summary Points}

Why carry out this study?

Glaucoma is a leading cause of irreversible blindness and ripasudil was the first Rho kinase inhibitor approved in Japan as antiglaucoma medication.

This study evaluated the long-term safety and effectiveness of ripasudil in Japanese patients with glaucoma or ocular hypertension in a real-word clinical setting, and was conducted as part of a risk management plan for safety monitoring activities.

\section{What was learned from the study?}

Ripasudil was safe and effective as an antiglaucoma medication with no new safety signals identified and significant reductions in intraocular pressure (IOP) maintained over 24 months of treatment.

The most common adverse drug reaction was blepharitis $(8.6 \%)$, which was also the most common reason for treatment discontinuation.

Multivariate analyses showed that blepharitis was significantly correlated with ripasudil treatment if patients were female, had comorbid or a previous history of blepharitis, or had a history of pollen or medication allergies.

\section{INTRODUCTION}

Glaucoma is a leading cause of irreversible blindness, with more than 70 million affected globally [1]; in Japan, $5.0 \%$ of individuals 40 years of age or more are estimated to have glaucoma [2]. This ocular disease is characterized by intraocular pressure (IOP)-associated optic neuropathy with progressive retinal ganglion cell death, resulting in irreversible visual field loss. To date, the proven therapeutic modality for the management of glaucoma has been reducing IOP [3], which slows disease progression and preserves quality of life $[4,5]$. A number of medications, including prostaglandin analogues, $\beta$-blockers, carbonic anhydrase inhibitors (CAIs), and $\alpha_{2}$-agonists, are approved and clinically used as antiglaucoma medications to reduce IOP. The IOP-lowering mechanisms of action of these medications include modifying the uveoscleral (unconventional) outflow route (e.g., prostaglandin analogues) and/or aqueous humor production (e.g., $\beta$-blockers) $[6,7]$. In contrast, Rho kinase (ROCK) inhibitors reduce IOP by a different mechanism from other antiglaucoma pharmacologic agents as they are the only treatment that directly targets the conventional aqueous humor outflow route, the main site of disease pathology in IOP elevation in glaucoma [8-12]. ROCK inhibitors modulate the conventional aqueous humor outflow by changing contractile properties, fibrotic activity, and permeability of cell components in the trabecular meshwork and Schlemm's canal [8, 13]. The first clinically available ROCK inhibitor was ripasudil, which was approved in Japan in September 2014 for the treatment of glaucoma and ocular hypertension $[12,14]$. As a part of the approval process for ripasudil in Japan, a large-scale post-marketing surveillance (PMS) study, titled ROCK-J, was conducted to evaluate the long-term safety and effectiveness of ripasudil in Japanese patients with glaucoma or ocular hypertension in a real-word clinical setting.

Interim analyses of the ROCK-J PMS study have shown that ripasudil safely and effectively lowered IOP after 3 and 12 months of treatment 
$[15,16]$. These interim analyses also revealed that the most common adverse drug reactions (ADRs) associated with ripasudil were blepharitis and conjunctivitis, causing discontinuation of treatment in some patients. Reported here are the final analyses of the ROCK-J PMS study, which were conducted at 24 months, and focused on the long-term IOP-lowering effects of ripasudil and potential baseline background factors for blepharitis during ripasudil treatment.

\section{METHODS}

\section{Study Design and Patients}

The design of this prospective, multicenter, open-label, observational PMS study has been described in detail previously $[15,16]$. Briefly, the study included ripasudil-naïve patients with glaucoma or ocular hypertension who were initiating treatment with ripasudil according to the Japanese approved indication between June 1, 2015 and April 30, 2017. Patients had to have an IOP measurement before ripasudil treatment was initiated and had to be enrolled within 14 days of initiating treatment. Patients were required to follow the dosing and administration instructions in the ripasudil package insert, which was to instill one drop of ripasudil twice a day and to wait at least 5 min between instillations when using other eye drops.

The study protocol was conducted in accordance with the Ministerial Ordinance on Good Post-Marketing Study Practice (GPSP, Ministry of Health, Labour and Welfare Ordinance No. 171, December 20, 2004) Japanese regulations. Under Japanese regulations, the protocols of PMS studies do not require review or approval by the ethics committee of the participating medical institutions or for collection of informed consent from patients (GPSP), unless such procedures were required by individual participating medical institutions. However, the study protocol was reviewed and approved by Pharmaceuticals and Medical Devices Agency, the Japanese Regulatory Authority, before initiation.

\section{Outcomes}

The primary safety endpoint was the incidence of ADRs, which was defined as an adverse event (AE) that was considered treatment-related by the investigator. ADRs were classified according to the International Council for Harmonisation of Technical Requirements for Pharmaceuticals for Human Use (ICH) Japanese Medical Dictionary for Regulatory Activities (MedDRA) version 22.1 and a key ADR of interest was blepharitis (including allergic response). Data on all allergic and/or inflammatory eye disorders were also collected. It is important to highlight that, as the event names were recorded by the physicians involved in the study, any recordings of "blepharitis" and "blepharitis allergic" were considered as a single ADR and described as blepharitis (including allergic response). The two types of blepharitis were grouped to remove uncertainty around the accurate clinical diagnosis of allergic blepharitis; this uncertainty arises because when doctors examine people with blepharitis in routine medical care, allergy tests are not often performed. This also applies to "conjunctivitis" and "conjunctivitis allergic", and "conjunctival hyperemia" or "ocular hyperemia".

The primary effectiveness endpoint was the least-squares mean (LSM) change in IOP from baseline to 24 months in all patients and in diagnostic subgroups. IOP was assessed in the affected eye, or in the eye with the highest baseline IOP if ripasudil was administered to both eyes. If both eyes had the same IOP at baseline, the right eye's data were used for evaluation. The secondary effectiveness endpoints were the change in IOP assessed by baseline IOP, the number of concomitant pharmaceutical components for glaucoma that the patients were receiving, and treatment initiation patterns.

Safety data were collected throughout the study, while IOP values were collected at 3,12 , 18 , and 24 months after initiating ripasudil. 


\section{Statistical Analysis}

Safety was assessed in the safety analysis set, which included all patients who had data from at least one visit to the clinic or hospital after initiating ripasudil treatment. Effectiveness was assessed in the effectiveness analysis set, which included all patients in the safety analysis set who had at least one measurement of IOP after initiating ripasudil treatment. If a patient changed glaucoma treatment after receiving ripasudil, or they underwent intraocular surgery (e.g., surgery for glaucoma or cataract, etc.), their data thereafter were excluded from the effectiveness analysis, because of the potentially confounding effects of these events on IOP.

Descriptive statistics (mean and standard deviation [SD]) were used to summarize continuous effectiveness variables, whereas categorical safety and effectiveness variables were summarized using number and proportion of patients. The Kaplan-Meier method was used to assess treatment continuation over the 24 months of follow-up (number, proportion of patients, and 95\% CIs) as well as the cumulative incidences of any ADR, allergy- and/or inflammation-related eye ADRs, blepharitis (including allergic response), and conjunctivitis (including allergic response). IOPs before treatment (baseline) were compared with IOPs after 24 months of follow-up (post-administration). The LSM, $\mathrm{SE}$, and $p$ values for the change from baseline to month 24 in IOP were estimated using a mixedeffects model for repeated measures (MMRM), with IOP measurements as the objective variable and indicator function (baseline and postadministration) as the fixed variables. The variance-covariance matrix had compound symmetry structure. MMRM was used to assess the overall change in IOP, as well as the change in IOP by disease type, type of secondary glaucoma, baseline IOP, the number of concomitant pharmaceutical components for glaucoma that the patients were receiving, and treatment initiation patterns.

Subgroup analyses and multivariate analyses were performed to find background factors for developing blepharitis with treatment. In the subgroup analyses, the number and proportion of patients experiencing blepharitis (including allergic response) by major patient characteristics were calculated, with differences tested using Fisher's exact or chi-squared tests. Multivariate regression analyses were performed using a Cox proportional-hazard model with the number of days from the start of treatment with ripasudil to the date of onset of the blepharitis as a response variable and major patient characteristics as candidates for explanatory variables. Sex (male, female), age ( $<65$ vs. $\geq 65$ to $<75$ years, $<65$ vs. $\geq 75$ years), presence or absence of medical history (blepharitis, conjunctivitis, diabetes mellitus, hypertension), presence or absence of allergic history (pollen, medications), number of concomitant pharmaceutical components for glaucoma, presence or absence of concomitant use of pharmaceutical components for glaucoma (prostaglandin analogues [including an ion channel opener], $\beta$ blockers, CAIs [topical and systemic formulations], $\alpha_{2}$-agonists, $\alpha_{1}$-blockers, parasympathomimetic drugs, and other glaucoma pharmaceutical agents), and presence or absence of concomitant pharmaceutical agents other than components for glaucoma (anti-allergic drugs, steroidal anti-inflammatory drugs, NSAIDs, drug treatments for dry eye, and antibiotics) were used in the subgroup analyses and were selected as explanatory variables for multivariate analyses. Multivariate analyses variables were selected using a stepwise method.

All statistical analyses were conducted using SAS Version 9.4 (SAS Institute, Japan).

\section{RESULTS}

\section{Patient Disposition and Characteristics}

Between June 1, 2015 and February 29, 2020, 3459 patients from 621 centers in Japan were registered in this PMS study. Data from 3434 patients were collected, and of these, 3374 were included in the safety analysis set (Fig. 1). The effectiveness analysis set included 3178 patients. The mean \pm SD observation period was $524.5 \pm 266.9$ days. At 24 months, $53.6 \%$ (95\% confidence interval [CI] 51.9-55.3) of patients continued to receive ripasudil treatment (Fig. 2). 
Overall, 1565 (46.4\%) patients discontinued the study. Reasons for study discontinuation included AEs ( $n=656 ; 19.4 \%)$, loss to follow-up (no visits recorded) or transfer to another hospital $(n=449 ; 13.3 \%)$, insufficient efficacy $(n=248 ; 7.4 \%)$, or patient's request, having no connection with the efficacy or safety of the drug $(n=107 ; 3.2 \%)$. The most common AEs leading to study discontinuation were blepharitis (including allergic response; $n=244 ; 7.2 \%$ ), conjunctivitis (including allergic response; $n=174 ; 5.2 \%$ ), and conjunctival hyperemia (including ocular hyperemia; $n=148 ; 4.4 \%$ ). Among 174 patients with conjunctivitis, 37 $(21.3 \%)$ patients discontinued treatment because of both conjunctivitis (including allergic response) and blepharitis (including allergic response); among 148 patients with conjunctival hyperemia, 17 (11.5\%) patients discontinued treatment because of both and conjunctival hyperemia (including ocular hyperemia) and blepharitis (including allergic response).

Patients in this study had a mean \pm SD age of $69.1 \pm 12.7$ years $(70.3 \%$ of patients were at least 65 years old) and $47.9 \%$ of patients were male (Table 1). Just under half of the patients included in this study had primary open-angle glaucoma (43.9\%), while $36.7 \%$ of patients had normal-tension glaucoma, $8.7 \%$ had secondary glaucoma (including exfoliation glaucoma [3.8\%], uveitis-associated glaucoma [1.7\%], steroid-induced glaucoma [1.0\%], and neovascular glaucoma $[0.7 \%]), 2.4 \%$ had primary angle-closure glaucoma, and $4.1 \%$ had ocular hypertension. At baseline, $20.1 \%$ had comorbid or a previous history of conjunctivitis while $4.1 \%$ had comorbid or a previous history of blepharitis. Regarding histories of eye surgery, $8.9 \%$ had prior surgery for glaucoma and $35.7 \%$ had prior surgery for cataracts. A total of $88.8 \%$ of patients had received previous glaucoma treatments and patients were receiving a mean of 2.1 previous and 2.2 concomitant pharmaceutical components for glaucoma.

\section{Safety}

Of the 3374 patients in the safety analysis set, ADRs were reported in 853 patients $(25.3 \%)$ with ripasudil over the 24-month follow-up period (Table 2). Of these patients experiencing an ADR, 742 patients $(87.0 \%)$ recovered or were in remission and $83(9.7 \%)$ did not recover. The

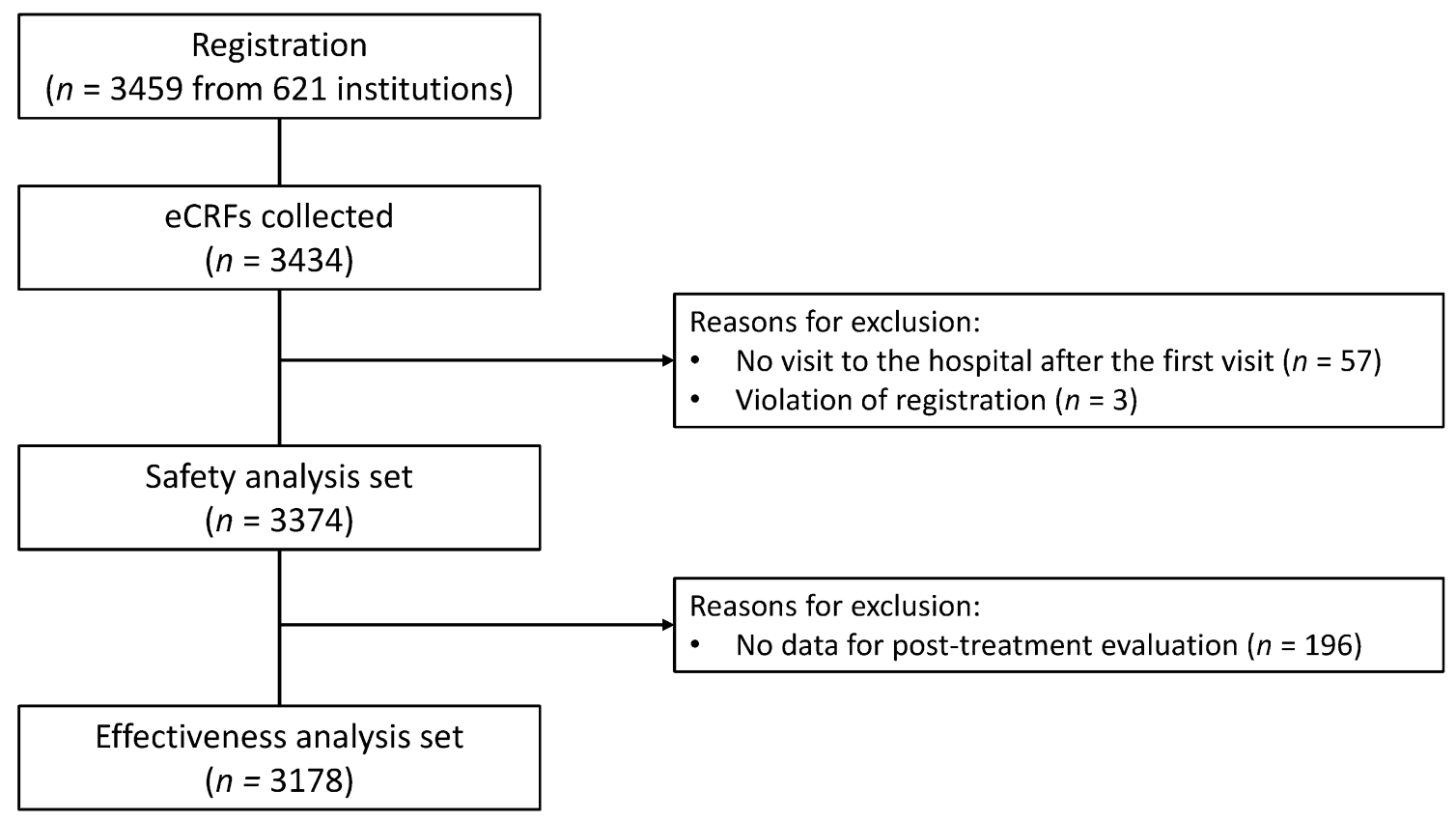

Fig. 1 Patient flow. eCRF electronic case report form, $n$ number of patients 


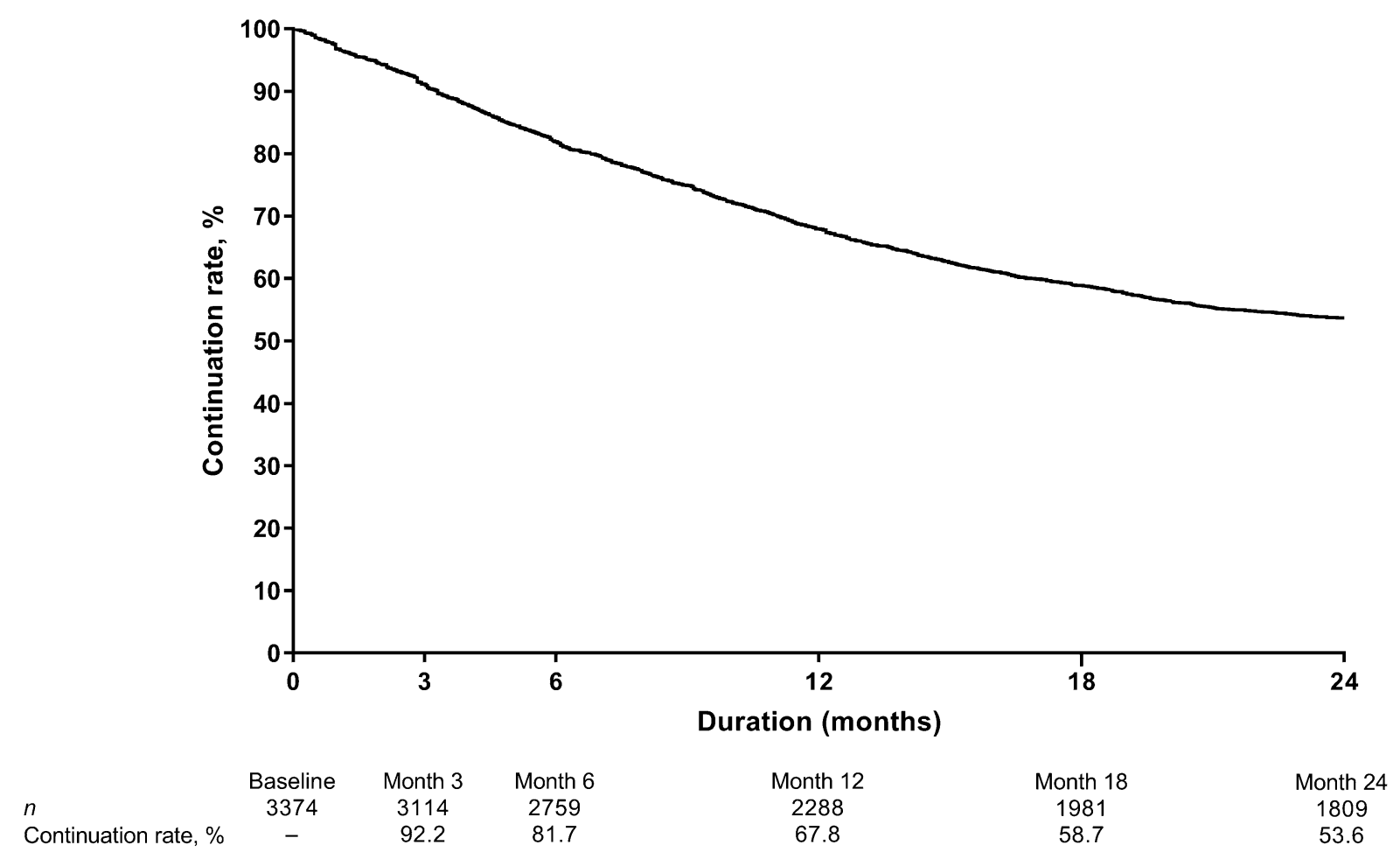

Fig. 2 Kaplan-Meier estimate of patient continuation over 24 months. $n$ number at risk

outcomes were unknown in 28 patients $(3.3 \%)$ experiencing an ADR.

The majority of ADRs reported were eye disorders, which occurred in $23.3 \%$ of patients; very few systemic ADRs were reported (Table 2; Table S1 in Supplementary Material). The most common eye-related ADRs were blepharitis (including allergic response; $n=290 ; 8.6 \%$ ), conjunctival hyperemia (including ocular hyperemia; $n=285 ; 8.5 \%$ ), conjunctivitis (including allergic response; $n=212 ; 6.3 \%$ ), and eye pruritus $(n=56 ; 1.7 \%)$. The cumulative incidence of ADRs was 7.2\%, 13.0\%, 21.7\%, $26.3 \%$, and $28.9 \%$ after $3,6,12,18$, and 24 months of follow-up, respectively (Fig. 3). In addition, the cumulative incidence of blepharitis (including allergic response), the most common ADR, was 1.0\%, 2.7\%, 7.2\%, 9.6\%, and $11.0 \%$ at $3,6,12,18$, and 24 months, respectively, and the cumulative incidence of conjunctivitis (including allergic response) was $1.0 \%, 3.0 \%, 5.4 \%, 7.1 \%$, and $7.8 \%$ at $3,6,12$, 18 , and 24 months, respectively (Fig. 3 ). The most common sites for allergy- and/or inflammation-related eye ADRs were the eyelids and conjunctiva (Table S2 in Supplementary Material). The cumulative incidence of allergyand/or inflammation-related eye ADRs was $2.7 \%, 6.9 \%, 14.1 \%, 17.8 \%$, and $19.9 \%$ after 3,6 , 12,18 , and 24 months of follow-up, respectively (Fig. 3).

Nine patients experienced 11 serious ADRs. Eye disorders included blepharitis (including allergic response; $n=5$ ), conjunctivitis (including allergic response; $n=2)$, cataract $(n=2)$, and conjunctival hyperemia (including ocular hyperemia; $n=1$ ); all 10 eye disorders improved or disappeared. The other ADR was cerebral infarction $(n=1)$, the outcome of which was unknown: it appeared a month after completion of treatment and was a recurrence in the patients who had medical history of this disease.

In the subgroup analysis, there was a significant difference in the incidence of blepharitis (including allergic response) with ripasudil treatment in female patients, older patients (age $\geq 65$ to $<75$ years or $\geq 75$ years), patients 
Table 1 Baseline characteristics

\begin{tabular}{|c|c|}
\hline Characteristic & $n=3374$ \\
\hline \multicolumn{2}{|l|}{ Sex } \\
\hline Male & $1617(47.9)$ \\
\hline Female & $1757(52.1)$ \\
\hline Mean \pm SD age, years & $69.1 \pm 12.7$ \\
\hline \multicolumn{2}{|l|}{ Age category } \\
\hline$<65$ years & $1002(29.7)$ \\
\hline$\geq 65$ to $<75$ years & $1156(34.3)$ \\
\hline$\geq 75$ years & $1216(36.0)$ \\
\hline \multicolumn{2}{|l|}{ Diagnosis } \\
\hline Ocular hypertension & $138(4.1)$ \\
\hline Primary open-angle glaucoma & $1480(43.9)$ \\
\hline Normal-tension glaucoma & $1237(36.7)$ \\
\hline Primary angle-closure glaucoma & $80(2.4)$ \\
\hline Secondary glaucoma & $295(8.7)$ \\
\hline Exfoliation glaucoma & $129(3.8)$ \\
\hline Uveitis-associated glaucoma & $56(1.7)$ \\
\hline Steroid-induced glaucoma & $33(1.0)$ \\
\hline Neovascular glaucoma & $23(0.7)$ \\
\hline Childhood glaucoma & $4(0.1)$ \\
\hline Other & $140(4.1)$ \\
\hline \multicolumn{2}{|l|}{ Disease history } \\
\hline $\begin{array}{l}\text { Comorbid or a previous history of } \\
\text { conjunctivitis }\end{array}$ & $677(20.1)$ \\
\hline $\begin{array}{l}\text { Comorbid or a previous history of } \\
\text { blepharitis }\end{array}$ & $139(4.1)$ \\
\hline Comorbid diabetes mellitus & $457(13.5)$ \\
\hline Comorbid hypertension & $814(24.1)$ \\
\hline History of pollen allergy & $331(9.8)$ \\
\hline History of medication allergy & $132(3.9)$ \\
\hline \multicolumn{2}{|l|}{ History of eye surgery } \\
\hline Glaucoma surgery & $299(8.9)$ \\
\hline Cataract surgery & $1203(35.7)$ \\
\hline
\end{tabular}

Table 1 continued

\begin{tabular}{ll}
\hline Characteristic & $n=3374$ \\
\hline $\begin{array}{l}\text { Previous pharmaceutical components for } \\
\text { glaucoma }\end{array}$ & $2997(88.8)$ \\
Mean \pm SD & $2.1 \pm 1.2$ \\
Prostaglandin analogues & $2657(78.8)$ \\
$\beta$-blockers & $2031(60.2)$ \\
CAIs (topical formulation) & $1373(40.7)$ \\
$\alpha_{2}$-agonists & $801(23.7)$ \\
\hline
\end{tabular}

All parameters are presented as number (proportion) of patients unless otherwise stated

CAIs carbonic anhydrase inhibitors, $S D$ standard deviation

Table 2 Adverse drug reactions occurring in at least 10 patients

\begin{tabular}{ll}
\hline ADRs, $\boldsymbol{n}(\%)$ & $\boldsymbol{n}=\mathbf{3 3 7 4}$ \\
\hline Any ADR & $853(25.3)$ \\
Blepharitis $^{\mathrm{a}}$ & $290(8.6)$ \\
Conjunctival hyperemia $^{\mathrm{b}}$ & $285(8.5)$ \\
Conjunctivitis $^{\mathrm{c}}$ & $212(6.3)$ \\
Eye pruritus & $56(1.7)$ \\
Punctate keratitis & $33(1.0)$ \\
Eye pain & $16(0.5)$ \\
Vision blurred & $13(0.4)$ \\
Eye irritation & $11(0.3)$ \\
Corneal erosion & $10(0.3)$ \\
Dry eye & $10(0.3)$ \\
\hline
\end{tabular}

ADRs occurring in fewer than 10 patients are listed in Table S1 (in Supplementary Material)

$A D R$ adverse drug reaction

${ }^{a}$ Including blepharitis allergic

bIncluding ocular hyperemia

${ }^{\mathrm{c}}$ Including conjunctivitis allergic 


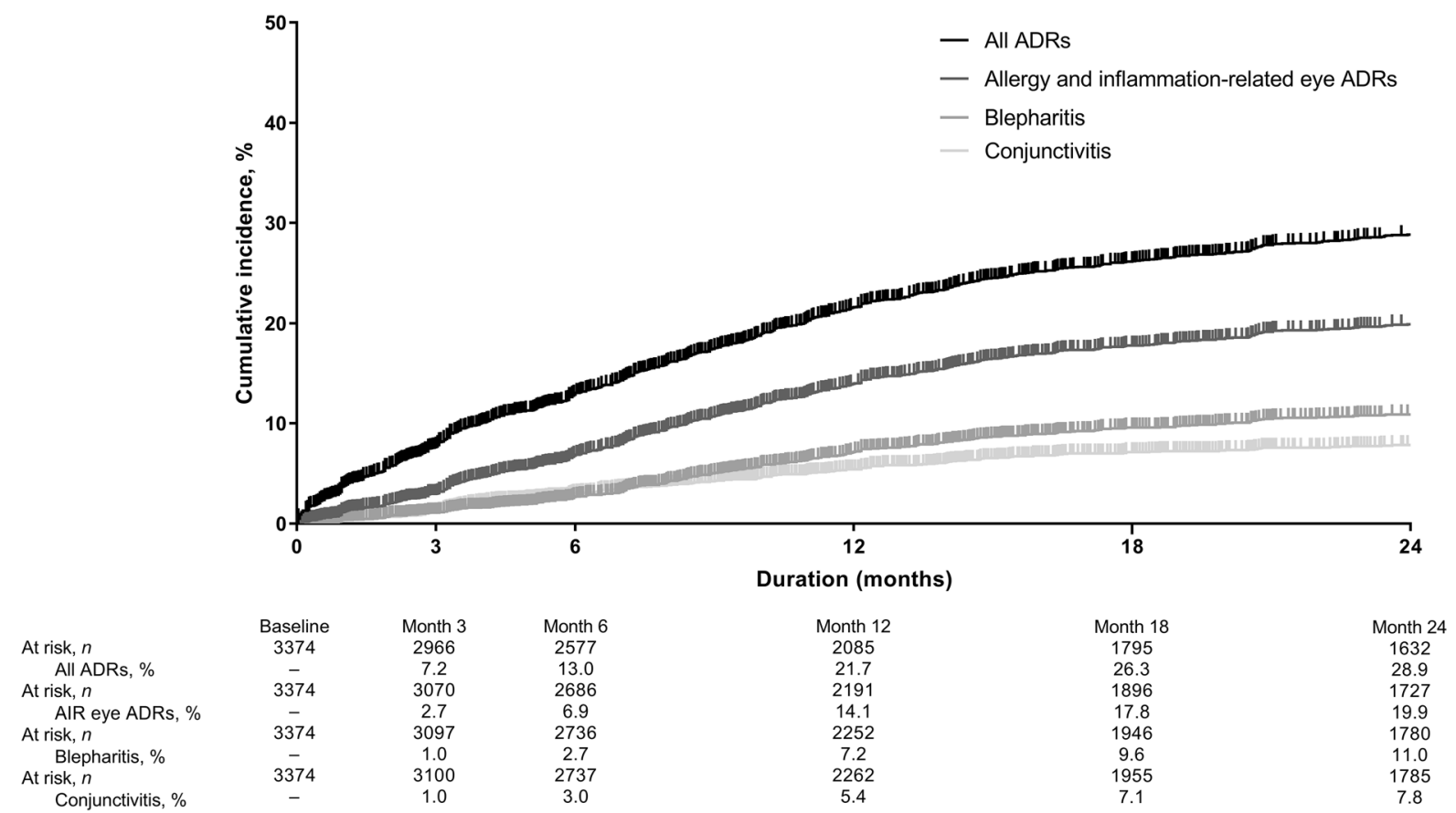

Fig. 3 Cumulative incidence of all adverse drug reactions, allergy- and/or inflammation-related eye ADRs, blepharitis (including allergic response), and conjunctivitis (including

with comorbid or a previous history of conjunctivitis or blepharitis, a history of pollen or medication allergy, a higher number of concomitant pharmaceutical components for glaucoma used, or those receiving concomitant drugs for glaucoma ( $\beta$-blockers, topical CAIs, or $\alpha_{2}$-agonists), or concomitant anti-allergic drugs (Table 3). However, there was no significant difference in the incidence of blepharitis (including allergic response) with ripasudil in patients with comorbid diabetes mellitus or hypertension, patients receiving other concomitant drugs for glaucoma (prostaglandin analogues, $\alpha_{1}$-blockers, parasympathomimetic drugs, systemic CAIs, or other pharmaceutical agents), or those receiving concomitant steroidal anti-inflammatory drugs, non-steroidal anti-inflammatory drugs (NSAIDs), antibiotics, or drug treatments for dry eye (Table 3). In the multivariate regression analyses, the following covariates were evaluated for potential association with the ADR blepharitis (including allergic response) during ripasudil treatment: sex, age, allergic response) over 24 months. ADR adverse drug reactions, AIR allergy- and/or inflammation-related, $n$ number at risk

comorbid or a previous history of blepharitis, history of pollen or medication allergy, drug treatments for dry eye, and the number of concomitant pharmaceutical components for glaucoma used. The multivariate analyses demonstrated that patients were more likely to experience the ADR blepharitis (including allergic response) with ripasudil treatment if they were female (hazard ratio [HR] 1.307; $p=0.040$ ), had comorbid or a previous history of blepharitis (HR 2.178; $p=0.001$ ), or had a history of pollen (HR 1.645; $p=0.003$ ) or medication (HR 2.276; $p<0.001$ ) allergies (Table 4).

\section{Effectiveness}

Overall, the least-squares mean (LSM) \pm standard error (SE) change from baseline in IOP after 24 months of ripasudil was $-2.6 \pm 0.1 \mathrm{mmHg}$ $(p<0.001)$; after 24 months, patients had a mean \pm SD IOP of $14.5 \pm 4.2 \mathrm{mmHg}$ (Fig. 4).

There was a significant decrease in IOP between baseline and 24 months in four types 
Table 3 Incidence of the adverse drug reaction blepharitis (including allergic response) with ripasudil treatment, by baseline characteristic

\begin{tabular}{|c|c|c|c|c|}
\hline Baseline characteristic & Category & $n$ & $\begin{array}{l}\text { Blepharitis, } \\
n(\%)\end{array}$ & $p$ value \\
\hline \multirow[t]{2}{*}{ Sex } & Male & 1617 & $114(7.1)$ & 0.002 \\
\hline & Female & 1757 & $176(10.0)$ & \\
\hline \multirow[t]{3}{*}{ Age } & $<65$ years & 1002 & $108(10.8)$ & 0.005 \\
\hline & $\begin{aligned} \geq & 65 \text { to } \\
& <75 \text { years }\end{aligned}$ & 1156 & $98(8.5)$ & \\
\hline & $\geq 75$ years & 1216 & $84(6.9)$ & \\
\hline \multirow[t]{2}{*}{ Comorbid or a previous history of conjunctivitis } & No & 2697 & $210(7.8)$ & 0.001 \\
\hline & Yes & 677 & $80(11.8)$ & \\
\hline \multirow[t]{2}{*}{ Comorbid or a previous history of blepharitis } & No & 3235 & $264(8.2)$ & $<0.001$ \\
\hline & Yes & 139 & $26(18.7)$ & \\
\hline \multirow[t]{2}{*}{ Comorbid diabetes mellitus } & No & 2917 & $254(8.7)$ & 0.591 \\
\hline & Yes & 457 & $36(7.9)$ & \\
\hline \multirow[t]{2}{*}{ Comorbid hypertension } & No & 2560 & $209(8.2)$ & 0.115 \\
\hline & Yes & 814 & $81(10.0)$ & \\
\hline \multirow[t]{2}{*}{ History of pollen allergy } & No & 2621 & $210(8.0)$ & $<0.001$ \\
\hline & Yes & 331 & $46(13.9)$ & \\
\hline \multirow[t]{2}{*}{ History of medication allergy } & No & 2820 & $232(8.2)$ & $<0.001$ \\
\hline & Yes & 132 & $24(18.2)$ & \\
\hline \multirow{5}{*}{$\begin{array}{l}\text { Number of concomitant pharmaceutical components for } \\
\text { glaucoma used }\end{array}$} & 0 & 348 & $33(9.5)$ & $<0.001$ \\
\hline & 1 & 729 & $70(9.6)$ & \\
\hline & 2 & 883 & $96(10.9)$ & \\
\hline & 3 & 915 & $72(7.9)$ & \\
\hline & $\geq 4$ & 499 & $19(3.8)$ & \\
\hline \multicolumn{5}{|l|}{ Concomitant pharmaceutical components for glaucoma } \\
\hline \multirow[t]{2}{*}{ Prostaglandin analogues } & No & 641 & $56(8.7)$ & 0.876 \\
\hline & Yes & 2733 & $234(8.6)$ & \\
\hline \multirow[t]{2}{*}{$\beta$-blockers } & No & 1251 & $126(10.1)$ & 0.022 \\
\hline & Yes & 2123 & $164(7.7)$ & \\
\hline \multirow[t]{2}{*}{ CAIs (topical formulation) } & No & 1917 & $188(9.8)$ & 0.004 \\
\hline & Yes & 1457 & $102(7.0)$ & \\
\hline
\end{tabular}


Table 3 continued

\begin{tabular}{|c|c|c|c|c|}
\hline Baseline characteristic & Category & $n$ & $\begin{array}{l}\text { Blepharitis, } \\
n(\%)\end{array}$ & $p$ value \\
\hline \multirow[t]{2}{*}{$\alpha_{2}$-agonists } & No & 2561 & $243(9.5)$ & $<0.001$ \\
\hline & Yes & 813 & $47(5.8)$ & \\
\hline \multirow[t]{2}{*}{$\alpha_{1}$-blockers } & No & 3334 & $288(8.6)$ & 0.576 \\
\hline & Yes & 40 & $2(5.0)$ & \\
\hline \multirow[t]{2}{*}{ Parasympathomimetic drugs } & No & 3340 & $290(8.7)$ & 0.113 \\
\hline & Yes & 34 & 0 & \\
\hline \multirow[t]{2}{*}{ CAIs (systemic formulation) } & No & 3291 & $286(8.7)$ & 0.318 \\
\hline & Yes & 83 & $4(4.8)$ & \\
\hline \multirow[t]{2}{*}{ Others } & No & 3221 & $282(8.8)$ & 0.141 \\
\hline & Yes & 153 & $8(5.2)$ & \\
\hline \multicolumn{5}{|c|}{ Other concomitant pharmaceutical agents } \\
\hline \multirow[t]{2}{*}{ Anti-allergic drugs } & No & 3117 & $253(8.1)$ & 0.001 \\
\hline & Yes & 257 & $37(14.4)$ & \\
\hline \multirow[t]{2}{*}{ Steroidal anti-inflammatory drugs } & No & 3030 & $259(8.6)$ & 0.761 \\
\hline & Yes & 344 & $31(9.0)$ & \\
\hline \multirow[t]{2}{*}{ NSAIDs } & No & 3144 & $269(8.6)$ & 0.716 \\
\hline & Yes & 230 & $21(9.1)$ & \\
\hline \multirow[t]{2}{*}{ Drug treatments for dry eye } & No & 2753 & $246(8.9)$ & 0.154 \\
\hline & Yes & 621 & $44(7.1)$ & \\
\hline \multirow[t]{2}{*}{ Antibiotics } & No & 3098 & $266(8.6)$ & 0.911 \\
\hline & Yes & 276 & $24(8.7)$ & \\
\hline
\end{tabular}

$C A I$ carbonic anhydrase inhibitor, NSAID non-steroidal anti-inflammatory drug

of glaucoma or ocular hypertension. The mean IOP reduction was $-3.9 \pm 0.4 \mathrm{mmHg}$ in 131 eyes with ocular hypertension $(p<0.001)$, $-2.9 \pm 0.1$ in 1446 eyes with primary openangle glaucoma $(p<0.001),-1.7 \pm 0.1$ in 1213 eyes with normal-tension glaucoma $(p<0.001),-4.2 \pm 0.5$ in 71 eyes with primary angle-closure glaucoma $(p<0.001)$, and $-3.8 \pm 0.4$ in 307 eyes with secondary glaucoma ( $p<0.001 ;$ Fig. 4$)$. Among patients with secondary glaucoma, ripasudil was associated with a significant decrease in IOP over the 24-month follow-up period in those with exfoliation glaucoma (LSM $\pm \mathrm{SE}$ change of $-2.8 \pm 0.4 \mathrm{mmHg} ; \quad p<0.001)$, uveitis-associated glaucoma $(-6.2 \pm 1.0 \mathrm{mmHg} ; p<0.001)$, and steroid-induced glaucoma $(-6.6 \pm 0.8 \mathrm{mmHg} ; \quad p<0.001)$, but not in those with neovascular glaucoma $(-1.0 \pm 1.9$ mmHg; $p=0.625$; Fig. 5$)$.

Significant reductions in IOP with ripasudil after 24 months were observed amongst all groups when categorized by baseline IOP (all $p<0.001$; Fig. 6). However, the magnitude of 
Table 4 Multivariate regression analyses of factors associated with experiencing the adverse drug reaction blepharitis (including allergic response) with ripasudil treatment

\begin{tabular}{lllr}
\hline Covariate & Comparison & HR $(95 \%$ CI $)$ & $\boldsymbol{p}$ value \\
\hline Sex & Female vs. male & $1.307(1.013-1.687)$ & 0.040 \\
Age & $\geq 65$ to $<75$ & $0.759(0.566-1.018)$ & 0.066 \\
& vs. $<65$ years & & \\
& $\geq 75$ vs. $<65$ years & $0.638(0.470-0.866)$ & 0.004 \\
Comorbid or a previous history of blepharitis & Yes vs. no & $2.178(1.352-3.508)$ & 0.001 \\
History of pollen allergy & Yes vs. no & $1.645(1.188-2.279)$ & 0.003 \\
History of medication allergy & Yes vs. no & $2.276(1.477-3.507)$ & $<0.001$ \\
Drug treatments for dry eye & Yes vs. no & $0.664(0.467-0.944)$ & 0.022 \\
Number of concomitant pharmaceutical components for & 1 vs. 0 & $0.855(0.554-1.318)$ & 0.478 \\
glaucoma used & 2 vs. 0 & $0.985(0.652-1.487)$ & 0.941 \\
& 3 vs. 0 & $0.637(0.413-0.981)$ & 0.041 \\
& $\geq 4$ vs. 0 & $0.346(0.191-0.626)$ & $<0.001$ \\
\hline
\end{tabular}

$C I$ confidence interval, $H R$ hazard ratio

the change was greater with increasing baseline IOP values, ranging from an LSM \pm SE change of $-0.6 \pm 0.1 \mathrm{mmHg}$ in patients with a baseline IOP of $\leq 15 \mathrm{mmHg}$ to a change of $-13.1 \pm 0.9 \mathrm{mmHg}$ in those with a baseline IOP of $>30 \mathrm{mmHg}$.

When categorized by the number of concomitant pharmaceutical components for glaucoma used, the change from baseline in IOP over the 24-month follow-up period was significant in all groups (all $p<0.001$; Fig. 7). The $\mathrm{LSM} \pm \mathrm{SE}$ change from baseline at 24 months ranged from $-2.5 \pm 0.2 \mathrm{mmHg}$ to $-2.6 \pm 0.2 \mathrm{mmHg}$ across patients who received zero to four or more concomitant pharmaceutical components for glaucoma.

Initiating treatment with ripasudil was associated with a significant reduction in IOP at 24 months regardless of the treatment initiation pattern (all $p<0.001$; Fig. S1 in Supplementary Material).

\section{DISCUSSION}

This is the final analysis of ROCK-J, a prospective, multicenter, open-label, observational,
PMS study that investigated the long-term safety and effectiveness of ripasudil in over 3000 patients with glaucoma or ocular hypertension in a real-world clinical practice setting in Japan. Over 24 months of follow-up, no new safety signals or major safety concerns were identified. Furthermore, the significant reductions in IOP that were apparent in the 3- and 12-month interim analyses were maintained at 24 months $[15,16]$.

At 24 months, the proportion of patients who had continued in the study was $53.6 \%$, which shows a gradual decline in the continuation rate from that observed in the 12-month interim analysis (70.3\%) [15]. The continuation rate found in the present analysis was similar to those of previous publications of real-world analyses of glaucoma medications in Japan and other countries [17-21]. Reasons for discontinuation from this study included AEs and loss to follow-up. Blepharitis (including allergic response) was the most common AE causing discontinuation of ripasudil, followed by conjunctivitis (including allergic response), and conjunctival hyperemia (including ocular hyperemia). 


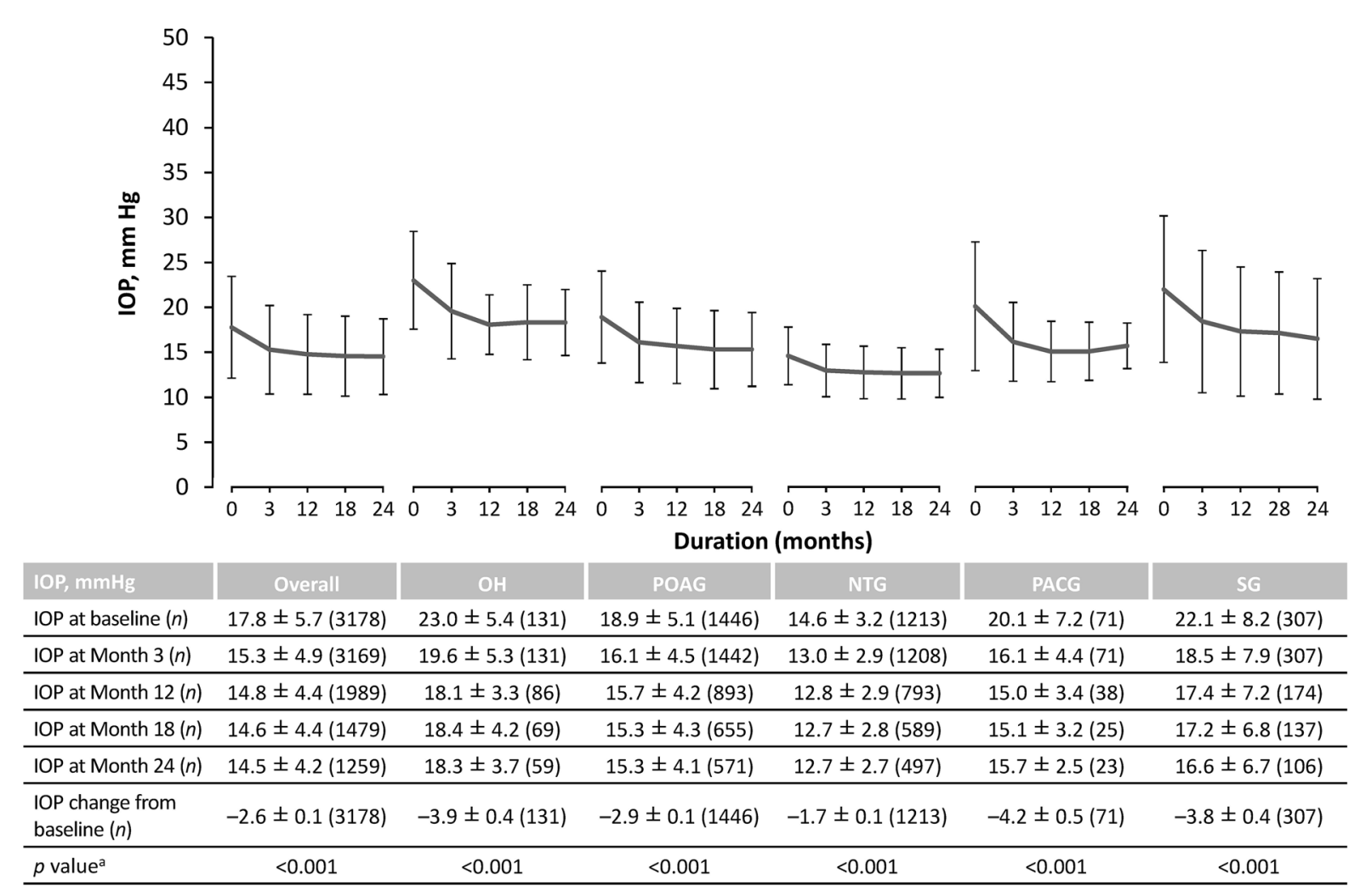

Fig. 4 Change in intraocular pressure overall and according to disease type. All values at specific timepoints are presented as mean \pm standard deviation, change from baseline values are presented as least-squares mean \pm

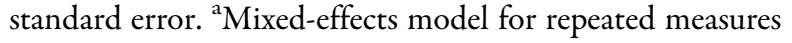

In the present study, blepharitis (including allergic response) was the most common ADR reported and the most common reason for discontinuation of ripasudil. Most ADRs, including blepharitis, conjunctivitis, and conjunctival hyperemia, were not considered serious and $87.0 \%$ of patients recovered or were in remission after developing an ADR. The incidences of these ADRs were low and only slightly elevated from those reported in the 12-month interim analysis of this PMS study [15]. The rates of discontinuation due to AEs were $7.2 \%$ for blepharitis (including allergic response), 5.2\% for conjunctivitis (including allergic response), and $4.4 \%$ for conjunctival hyperemia (including ocular hyperemia). Among patients with conjunctivitis $(n=174)$ or conjunctival hyperemia $(n=148)$, the reasons for discontinuation were for overall change from baseline. IOP intraocular pressure, $n$ number of patients, NTG normal-tension glaucoma, $\mathrm{OH}$ ocular hypertension, PACG primary angle-closure glaucoma, POAG primary open-angle glaucoma, SG secondary glaucoma

blepharitis and conjunctivitis in $21.3 \%$ and blepharitis and conjunctival hyperemia in $11.5 \%$ of cases, respectively. To date, only a few small-scale clinical studies have investigated the long-term safety and effectiveness of ripasudil over 24 months in patients with glaucoma $[22,23]$. While these studies did not report the individual incidences of the aforementioned ADRs, in one study, $15.7 \%, 9.0 \%$, and $5.4 \%$ of patients discontinued treatment because of blepharitis, conjunctival hyperemia, and allergic conjunctivitis, respectively [22], while in the other study, $25.2 \%$ and $7.8 \%$ of patients discontinued treatment because of blepharitis and prolonged hyperemia [23], which are higher discontinuation rates than those seen in this PMS study. From a clinical viewpoint, these small-scale clinical studies, as well as the results 


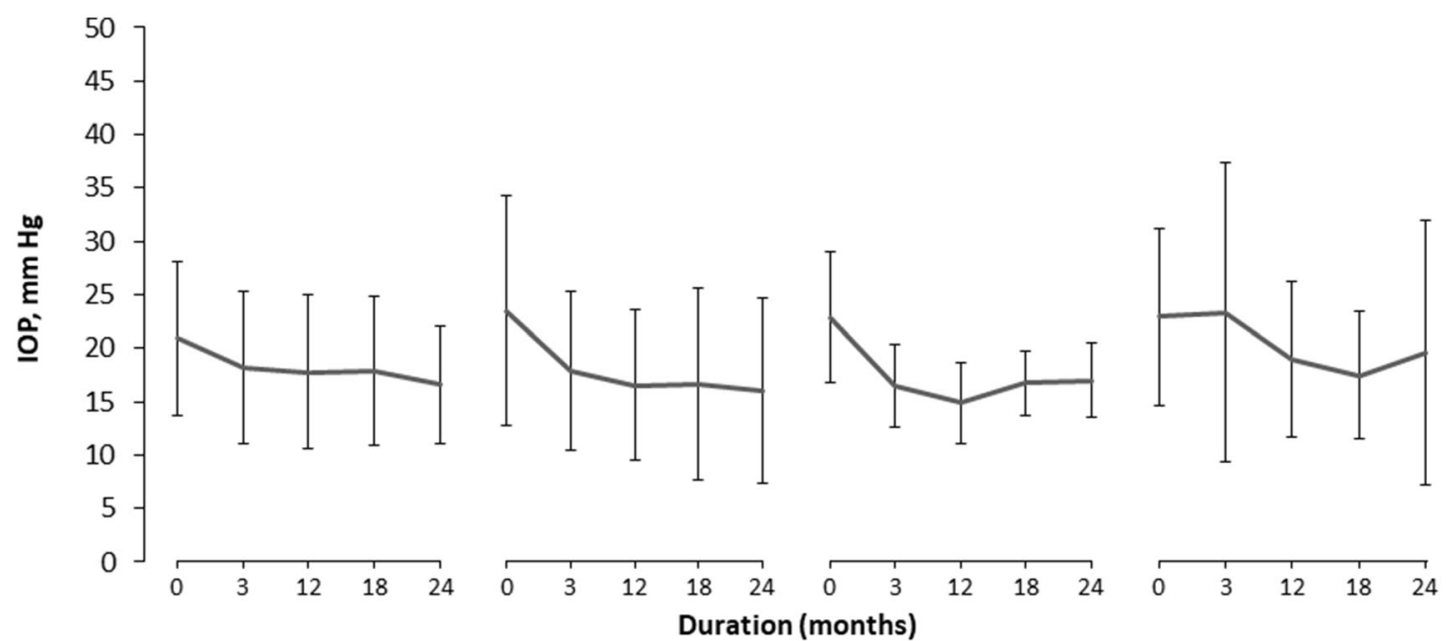

\begin{tabular}{l|c|cc|c}
\hline IOP, $\mathrm{mmHg}$ & $\begin{array}{c}\text { Exfoliation } \\
\text { Elaucoma }\end{array}$ & $\begin{array}{c}\text { Uveitis-associated } \\
\text { glaucoma }\end{array}$ & $\begin{array}{c}\text { Steroid-induced } \\
\text { glaucoma }\end{array}$ & $\begin{array}{c}\text { Neovascular } \\
\text { glaucoma }\end{array}$ \\
\hline IOP at baseline $(n)$ & $20.9 \pm 7.2(147)$ & $23.5 \pm 10.7(57)$ & $22.9 \pm 6.1(30)$ & $23.0 \pm 8.3(22)$ \\
\hline IOP at Month $3(n)$ & $18.1 \pm 7.1(147)$ & $17.8 \pm 7.5(57)$ & $16.5 \pm 3.9(30)$ & $23.4 \pm 14.0(22)$ \\
\hline IOP at Month 12 $(n)$ & $17.8 \pm 7.3(84)$ & $16.6 \pm 7.1(31)$ & $14.9 \pm 3.8(18)$ & $19.0 \pm 7.3(12)$ \\
\hline IOP at Month 18 $(n)$ & $17.8 \pm 7.0(65)$ & $16.6 \pm 9.0(28)$ & $16.8 \pm 3.1(14)$ & $17.5 \pm 6.1(10)$ \\
\hline IOP at Month 24 $(n)$ & $16.6 \pm 5.6(47)$ & $16.0 \pm 8.7(24)$ & $17.1 \pm 3.5(13)$ & $19.6 \pm 12.4(7)$ \\
\hline $\begin{array}{l}\text { IOP change from } \\
\text { baseline }(n)\end{array}$ & $-2.8 \pm 0.4(147)$ & $-6.2 \pm 1.0(57)$ & $-6.6 \pm 0.8(30)$ & $-1.0 \pm 1.9(22)$ \\
\hline$p$ value & $<0.001$ & $<0.001$ & $<0.001$ & 0.625 \\
\hline
\end{tabular}

Fig. 5 Change in intraocular pressure in patients according to the type of secondary glaucoma. All values at specific timepoints are presented as mean \pm standard deviation, change from baseline values are presented as least-squares mean \pm standard error. ${ }^{a}$ Mixed-effects model for repeated measures for overall change from baseline. IOP intraocular pressure, $n$ number of patients

stabilized thereafter, suggesting that patients are at the highest risk of developing blepharitis after receiving ripasudil for between 6 and 12 months.

As blepharitis was the most common $\mathrm{AE}$, and the most common AE leading to study discontinuation, background factors for developing blepharitis with ripasudil treatment were assessed. Multivariate regression analyses revealed that patients in this PMS study were approximately two times more likely to develop blepharitis if they had comorbid or a previous history of blepharitis (HR 2.178; 95\% CI $1.352-3.508 ; p=0.001$ vs. patients without comorbid or a previous history of blepharitis), if they had a history of allergies to medications (HR 2.276; 95\% CI 1.477-3.507; $p<0.001$ vs. patients without a history of allergies to 


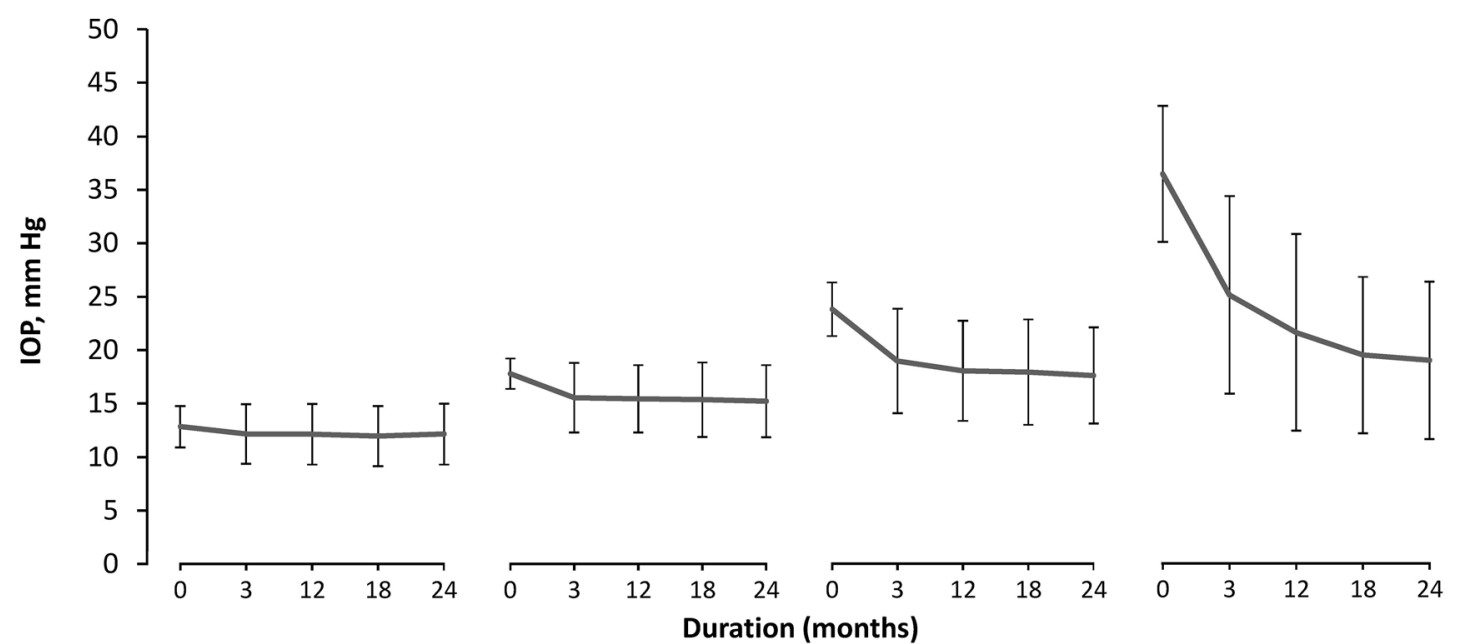

\begin{tabular}{lcccc} 
IOP, $\mathrm{mmHg}$ & $\leq 15 \mathrm{mmHg}$ & $>15$ to $\leq 20 \mathrm{mmHg}$ & $>20$ to $\leq 30 \mathrm{mmHg}$ & $>30 \mathrm{mmHg}$ \\
\hline IOP at baseline $(n)$ & $12.9 \pm 1.9(1220)$ & $17.8 \pm 1.4(1153)$ & $23.9 \pm 2.5(717)$ & $36.6 \pm 6.4(88)$ \\
\hline IOP at Month 3 $(n)$ & $12.2 \pm 2.8(1218)$ & $15.6 \pm 3.3(1149)$ & $19.0 \pm 4.9(715)$ & $25.2 \pm 9.3(87)$ \\
\hline IOP at Month $12(n)$ & $12.1 \pm 2.8(808)$ & $15.4 \pm 3.2(732)$ & $18.1 \pm 4.7(406)$ & $21.7 \pm 9.2(43)$ \\
\hline IOP at Month 18 $(n)$ & $12.0 \pm 2.8(616)$ & $15.4 \pm 3.5(522)$ & $18.0 \pm 5.0(312)$ & $19.6 \pm 7.4(29)$ \\
\hline IOP at Month 24 $(n)$ & $12.2 \pm 2.8(518)$ & $15.2 \pm 3.4(459)$ & $17.6 \pm 4.5(256)$ & $19.1 \pm 7.4(26)$ \\
\hline $\begin{array}{l}\text { IOP change from } \\
\text { baseline }(n)\end{array}$ & $-0.6 \pm 0.1(1220)$ & $-2.3 \pm 0.1(1153)$ & $-5.2 \pm 0.2(717)$ & $-13.1 \pm 0.9(88)$ \\
\hline$p$ value $^{\mathrm{a}}$ & $<0.001$ & $<0.001$ & $<0.001$ & $<0.001$ \\
\hline
\end{tabular}

Fig. 6 Change in intraocular pressure in patients according to baseline intraocular pressure values. All values at specific timepoints are presented as mean \pm standard deviation, change from baseline values are presented as

medications) or if they had a history of allergies to pollen (HR 1.645; 95\% CI 1.188-2.279; $p=0.003)$. This was also seen in a report by Saito and colleagues, who showed that patients with history of allergic reactions to other topical glaucoma medications were more likely to experience blepharitis than those without an allergic history (HR 3.599; 95\% CI 1.416-9.152; $p=0.007$ ) [23]. Taken together, these results suggest that caution should be exercised when administering ripasudil to patients with an allergic history.

Multivariate regression analyses of this PMS study also demonstrated that female patients were more likely to develop blepharitis (including allergic response) than men. This may be due to multiple factors. Firstly, as blepharitis can result in cosmetic changes, it is more likely that women would report this to their least-squares mean \pm standard error. ${ }^{a}$ Mixed-effects model for repeated measures for overall change from baseline. IOP intraocular pressure, $n$ number of patients

physicians than men do. Furthermore, endocrine differences between men and women may influence this immune response.

Multivariate regression analyses also indicated that age (75 years or more), the number of concomitant pharmaceutical components for glaucoma used, and concomitant treatment with drugs for dry eye reduced the risk of a patient developing blepharitis. It is likely that elderly patients were less immunocompetent than those aged less than 65 years, which would reduce the risk of them developing blepharitis (including allergic response). As for the number of concomitant pharmaceutical components for glaucoma used, a study entry bias may exist, by which patients who were already receiving and tolerating multiple treatments for glaucoma were more likely to be selected to initiate treatment with ripasudil than those who were 


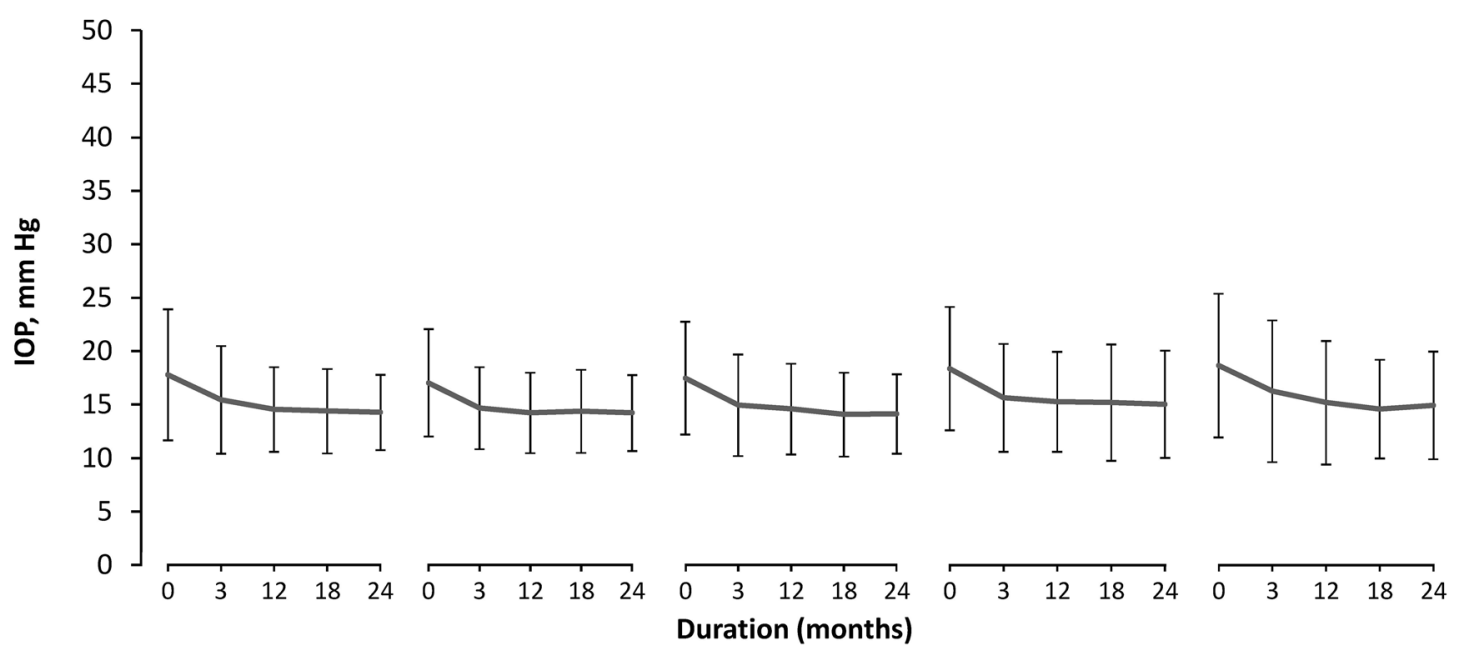

\begin{tabular}{lccccc} 
IOP, $\mathrm{mmHg}$ & \multicolumn{1}{c}{0} & 1 & 3 & 2 \\
IOP at baseline $(n)$ & $17.8 \pm 6.1(395)$ & $17.0 \pm 5.0(801)$ & $17.5 \pm 5.3(869)$ & $18.4 \pm 5.8(775)$ & $18.7 \pm 6.8(338)$ \\
\hline IOP at Month $3(n)$ & $15.5 \pm 5.0(393)$ & $14.7 \pm 3.8(801)$ & $15.0 \pm 4.8(864)$ & $15.7 \pm 5.1(773)$ & $16.3 \pm 6.7(338)$ \\
\hline IOP at Month $12(n)$ & $14.6 \pm 3.9(224)$ & $14.2 \pm 3.7(510)$ & $14.7 \pm 4.3(534)$ & $15.3 \pm 4.7(510)$ & $15.2 \pm 5.8(211)$ \\
\hline IOP at Month $18(n)$ & $14.4 \pm 3.9(167)$ & $14.4 \pm 3.9(386)$ & $14.1 \pm 3.9(384)$ & $15.3 \pm 5.5(381)$ & $14.6 \pm 4.6(161)$ \\
\hline IOP at Month 24 $(n)$ & $14.3 \pm 3.5(153)$ & $14.2 \pm 3.5(322)$ & $14.2 \pm 3.7(322)$ & $15.1 \pm 5.0(322)$ & $15.0 \pm 5.0(140)$ \\
\hline $\begin{array}{l}\text { IOP change from } \\
\text { baseline }(n)\end{array}$ & $-2.5 \pm 0.2(395)$ & $-2.5 \pm 0.1(801)$ & $-2.6 \pm 0.1(869)$ & $-2.6 \pm 0.1(775)$ & $-2.6 \pm 0.2(338)$ \\
\hline$p$ value $^{\mathrm{a}}$ & $<0.001$ & $<0.001$ & $<0.001$ & $<0.001$ & $<0.001$ \\
\hline
\end{tabular}

Fig. 7 Change in intraocular pressure in patients according to the number of concomitant pharmaceutical components for glaucoma used. All values at specific timepoints are presented as mean \pm standard deviation,

not. Also, the washout effect may have occurred in patients who were receiving more concomitant pharmaceutical components for glaucoma. This can also possibly explain how the concomitant treatment with drugs for dry eye was associated with a lower risk of developing blepharitis. As dry eye treatments are typically administered multiple times a day, the washout effect on the eye surface might mean that the exposure to ripasudil was less than in patients not receiving dry eye treatment. We recommend that physicians carefully monitor patients who regularly used dry eye medications for potential washout effect.

No corneal disorders of concern were seen in this PMS study; none of the patients receiving ripasudil developed corneal verticillata, an ADR that has a well-known association with netarsudil (another ROCK inhibitor available for the change from baseline values are presented as least-squares mean \pm standard error. ${ }^{a}$ Mixed-effects model for repeated measures for overall change from baseline. IOP intraocular pressure, $n$ number of patients

treatment of glaucoma) $[25,26]$. Furthermore, there were no ADRs suggestive of corneal endothelial dysfunction (e.g., corneal opacification, corneal edema, or bullous keratopathy) with ripasudil over the 24-month follow-up period. While some investigations have shown that ripasudil causes a temporary morphologic change of corneal endothelial cells in humans and animals [27, 28], this has not translated into an increased risk of developing corneal disorders in patients with glaucoma receiving ripasudil $[24,29,30]$. Furthermore, ROCK inhibitors, such as Y-27632 and ripasudil, are regarded as a having regenerative effect on diseased corneal endothelial cells [31, 32]. Taking these points into consideration, the final results of this PMS study show that the clinical properties of ripasudil are different from those of 
netarsudil, which is reflected by the different safety profile on the cornea.

In line with other clinical trials of ripasudil $[24,29,30,33]$ and previous interim analyses of this PMS study $[15,16]$, IOP was significantly reduced with ripasudil in this final analysis of the PMS study. The change was apparent at the 3-month assessment and maintained until 24 months. The significant reduction in IOP was seen when patients were categorized by type of disease, baseline IOP values, and the number of concomitant pharmaceutical components for glaucoma, with the exception of patients with neovascular glaucoma. In all of the other subtypes of secondary glaucoma assessed in this PMS study, large changes in IOP were observed over 24 months, supporting previous research that has shown that ripasudil is effective in patients with exfoliation, uveitisassociated, and steroid-induced glaucoma [34-36]. In the pathogenesis of neovascular glaucoma, fibrovascular proliferative tissue seriously damages the conventional aqueous humor outflow [37], and, as such, this may explain why ripasudil did not significantly reduce IOP in these patients. However, it is also important to note that the subgroup of patients with neovascular glaucoma in this PMS study was small $(n=22)$, and was probably underpowered to detect a significant treatment difference.

In this PMS study, $88.8 \%$ of patients had received previous glaucoma treatments and patients were receiving a mean of 2.2 concomitant pharmaceutical components for glaucoma at the start of treatment with ripasudil. Despite this, the results of this PMS study showed that adding ripasudil to treatment with four or more other pharmaceutical components for glaucoma still resulted in a significant decrease in IOP, which was maintained over the 24-month follow-up period. These results indicate that ripasudil provides a valuable add-on treatment option in daily clinical practice, most likely because of its distinct mechanism of action.

This study has a number of limitations, which are intrinsic with open-label, non-comparative studies. While the prospective collection of daily medical information from a large number of patients is a major strength of this observational study, limitations include the variation in treatment plans, such as when to add drug treatment, and target IOP, which are at the discretion of the physician. Also, the ADRs collected are the result of subjective evaluation by the physician, so we decided to group blepharitis with allergic blepharitis. Allergy tests are not often performed when physicians examine people with blepharitis in routine medical care. As a result of this and the fact that our study protocol did not require allergic blepharitis to be confirmed with an allergy test, we considered it was appropriate to group blepharitis with allergic blepharitis to remove uncertainty around the accurate clinical diagnosis of allergic blepharitis. This reduced the chance of the authors deriving a conclusion from a condition that had not been accurately diagnosed. In addition, the absence of a control group can lead to selection bias in the patient population and a lack of control for unknown confounders (such as laser surgery or other surgery) in evaluating safety and effectiveness. Some of the subgroup analyses were performed in smaller numbers of patients. While the outcomes did reach statistical significance, similar studies in larger numbers of patients with the various secondary glaucoma diagnoses would help to confirm the current findings. Furthermore, the observational nature of the study may lead to a regression to the mean. Finally, as this study only included patients from Japan, the generalizability of the outcomes to individuals of other ethnicities is limited.

\section{CONCLUSION}

In patients with glaucoma or ocular hypertension, treatment with ripasudil in a real-world clinical setting was not associated with any significant safety concerns after 24 months of follow-up. In addition, ripasudil was associated with stable and sufficient IOP-lowering effects in combination with other antiglaucoma medications. This suggests that ripasudil has an additive effect, and highlights that it is a useful treatment option for patients with glaucoma or ocular hypertension. 


\section{ACKNOWLEDGEMENTS}

The authors thank all clinicians and patients for their involvement and contribution to the study.

Funding. This study, and the journal's Rapid Service and Open Access fees associated with publication, were funded by Kowa Company, Ltd, Tokyo, Japan.

Medical Writing Assistance. The authors also thank Yoshiko Okamoto, PhD, and Simone Tait, of inScience Communications, Springer Healthcare, for writing the outline and first draft of the manuscript, respectively. This medical writing assistance was funded by Kowa Company, Ltd.

Authorship. All named authors meet the International Committee of Medical Journal Editors (ICMJE) criteria for authorship for this article, take responsibility for the integrity of the work as a whole, and have given their approval for this version to be published.

Author Contributions. All authors participated in study concept, study design (with recruitment of subjects and data collection was carried out by Kowa Company, Ltd.), data analysis and interpretation (having full access to all study data), and take responsibility for the integrity of the data and accuracy of the data analysis. The authors contributed to the first draft of the manuscript and subsequent revisions, and read and approved the final manuscript for submission.

Disclosures. Hidenobu Tanihara has received research funding from Kowa, Santen Pharmaceutical, and Senju Pharmaceutical, consulting fees from Kowa, Aerie Pharmaceuticals, Inc., Santen Pharmaceutical, and Wakamoto Pharmaceutical, and honoraria for lectures from Kowa, Senju Pharmaceutical, Santen Pharmaceutical, Otsuka Pharma Co. Ltd., Pfizer, Rohto Pharmaceutical, Wakamoto Pharmaceutical, HOYA Corporation, Mitsubishi Tanabe Pharma, Glaukos Japan, and Novartis Pharma. Takahiko Kakuda, Tetsuro Sano,
Takashi Kanno, and Yuji Kurihara are employees of Kowa Co., Ltd.

Compliance with Ethics Guidelines. This study is a multicenter, open-label, prospective, non-interventional study and was conducted in accordance with the Ministerial Ordinance on Good Post-Marketing Study Practice (Ministry of Health, Labour and Welfare Ordinance No. 171, December 20, 2004) Japanese guidelines. The study protocol was reviewed and approved by Pharmaceuticals and Medical Devices Agency, the Japanese Regulatory Authority, before initiation. This study did not undergo review by the ethics committee of the participating medical institutions or the informed consent procedure; according to Japanese regulations, approval by the ethics committee of each participating medical institution and informed consent from individual patients are not needed for post-marketing surveillance.

Data Availability. The datasets generated during and/or analyzed during the current study are available from the corresponding author on reasonable request.

Open Access. This article is licensed under a Creative Commons Attribution-NonCommercial 4.0 International License, which permits any non-commercial use, sharing, adaptation, distribution and reproduction in any medium or format, as long as you give appropriate credit to the original author(s) and the source, provide a link to the Creative Commons licence, and indicate if changes were made. The images or other third party material in this article are included in the article's Creative Commons licence, unless indicated otherwise in a credit line to the material. If material is not included in the article's Creative Commons licence and your intended use is not permitted by statutory regulation or exceeds the permitted use, you will need to obtain permission directly from the copyright holder. To view a copy of this licence, visit http://creativecommons.org/licenses/bync/4.0/. 


\section{REFERENCES}

1. Quigley HA, Broman AT. The number of people with glaucoma worldwide in 2010 and 2020. Br J Ophthalmol. 2006;90:262-7.

2. Yamamoto T, Iwase A, Araie M, et al. The Tajimi Study report 2: prevalence of primary angle closure and secondary glaucoma in a Japanese population. Ophthalmology. 2005;112:1661-9.

3. Boland MV, Ervin AM, Friedman DS, et al. Comparative effectiveness of treatments for open-angle glaucoma: a systematic review for the US Preventive Services Task Force. Ann Intern Med. 2013;158: 271-9.

4. Quigley HA. Glaucoma. Lancet. 2011;377:1367-77.

5. Weinreb RN, Aung T, Medeiros FA. The pathophysiology and treatment of glaucoma: a review. JAMA. 2014;311:1901-11.

6. Cvenkel B, Kolko M. Current medical therapy and future trends in the management of glaucoma treatment. J Ophthalmol. 2020;2020:6138132.

7. Bucolo C, Salomone S, Drago F, et al. Pharmacological management of ocular hypertension: current approaches and future prospective. Curr Opin Pharmacol. 2013;13:50-5.

8. Honjo M, Tanihara H. Impact of the clinical use of ROCK inhibitor on the pathogenesis and treatment of glaucoma. Jpn J Ophthalmol. 2018;62:109-26.

9. Honjo $\mathrm{M}$, Tanihara $\mathrm{H}$, Inatani $\mathrm{M}$, et al. Effects of Rho-associated protein kinase inhibitor Y-27632 on intraocular pressure and outflow facility. Invest Ophthalmol Vis Sci. 2001;42:137-44.

10. Rao PV, Deng P-F, Kumar J, et al. Modulation of aqueous humor outflow facility by the Rho kinasespecific inhibitor Y-27632. Invest Ophthalmol Vis Sci. 2001;42:1029-37.

11. Tanihara $\mathbf{H}$, Inatani $\mathbf{M}$, Honjo $\mathbf{M}$, et al. Intraocular pressure-lowering effects and safety of topical administration of a selective ROCK inhibitor, SNJ1656, in healthy volunteers. Arch Ophthal. 2008;126:309-15.

12. Garnock-Jones KP. Ripasudil: first global approval. Drugs. 2014;74:2211-5.

13. Moshirfar M, Parker L, Birdsong OC, et al. Use of Rho kinase inhibitors in ophthalmology: a review of the literature. Med Hypothesis Discov Innov Ophthalmol. 2018;7:101-11.
14. Inoue $T$, Tanihara $H$. Ripasudil hydrochloride hydrate: targeting Rho kinase in the treatment of glaucoma. Expert Opin Pharmacother. 2017;18: 1669-73.

15. Tanihara H, Kakuda T, Sano T, et al. Safety and efficacy of ripasudil in Japanese patients with glaucoma or ocular hypertension: 12-month interim analysis of ROCK-J, a post-marketing surveillance study. BMC Ophthalmol. 2020;20:275.

16. Tanihara H, Kakuda T, Sano T, et al. Safety and efficacy of ripasudil in Japanese patients with glaucoma or ocular hypertension: 3-month interim analysis of ROCK-J, a post-marketing surveillance study. Adv Ther. 2019;36:333-43.

17. Kashiwagi K, Furuya T. Persistence with topical glaucoma therapy among newly diagnosed Japanese patients. Jpn J Ophthalmol. 2014;58:68-74.

18. Rouland JF, Le Pen C, Benhaddi H, et al. Naturalistic, prospective study of glaucoma and ocular hypertension treatment in France: strategies, clinical outcomes, and costs at 2 years. Eur J Ophthalmol. 2005;15:562-80.

19. Goldberg I, Li XY, Selaru P, et al. A 5-year, randomized, open-label safety study of latanoprost and usual care in patients with open-angle glaucoma or ocular hypertension. Eur J Ophthalmol. 2008;18: 408-16.

20. Zimmerman TJ, Hahn SR, Gelb L, et al. The impact of ocular adverse effects in patients treated with topical prostaglandin analogs: changes in prescription patterns and patient persistence. J Ocul Pharmacol Ther. 2009;25:145-52.

21. Hwang DK, Liu CJ, Pu CY, et al. Persistence of topical glaucoma medication: a nationwide population-based cohort study in Taiwan. JAMA Ophthalmol. 2014;132:1446-52.

22. Maruyama Y, Ikeda Y, Mori K, et al. Safety and efficacy of long-term ripasudil $0.4 \%$ instillation for the reduction of intraocular pressure in Japanese open-angle glaucoma patients. J Ocul Pharmacol Ther. 2020;36:229-33.

23. Saito H, Kagami S, Mishima K, et al. Long-term side effects including blepharitis leading to discontinuation of ripasudil. J Glaucoma. 2019;28:289-93.

24. Tanihara H, Inoue T, Yamamoto $\mathrm{T}$, et al. One-year clinical evaluation of $0.4 \%$ ripasudil (K-115) in patients with open-angle glaucoma and ocular hypertension. Acta Ophthalmol. 2016;94:e26-34.

25. Serle JB, Katz LJ, McLaurin E, et al. Two phase 3 clinical trials comparing the safety and efficacy of netarsudil to timolol in patients with elevated 
intraocular pressure: Rho kinase elevated IOP treatment trial 1 and 2 (ROCKET-1 and ROCKET-2). Am J Ophthalmol. 2018;186:116-27.

26. Kahook MY, Serle JB, Mah FS, et al. Long-term safety and ocular hypotensive efficacy evaluation of netarsudil ophthalmic solution: Rho kinase elevated IOP treatment trial (ROCKET-2). Am J Ophthalmol. 2019;200:130-7.

27. Maruyama Y, Ikeda Y, Mori K, et al. Morphological change and recovery of corneal endothelial cells after rho-associated protein kinase inhibitor eyedrop (ripasudil 0.4\%) instillation. Br J Ophthalmol. 2021;105:169-73.

28. Okumura N, Okazaki Y, Inoue R, et al. Rho-associated kinase inhibitor eye drop (ripasudil) transiently alters the morphology of corneal endothelial cells. Invest Ophthalmol Vis Sci. 2015;56:7560-7.

29. Tanihara $H$, Inoue $T$, Yamamoto $T$, et al. Phase 1 clinical trials of a selective Rho kinase inhibitor, K-115. JAMA Ophthalmol. 2013;131:1288-95.

30. Tanihara $H$, Inoue $\mathrm{T}$, Yamamoto $\mathrm{T}$, et al. Phase 2 randomized clinical study of a Rho kinase inhibitor, $\mathrm{K}-115$, in primary open-angle glaucoma and ocular hypertension. Am J Ophthalmol. 2013;156:731-6 e2.

31. Schlötzer-Schrehardt U, Zenkel M, Strunz M, et al. Potential functional restoration of corneal endothelial cells in fuchs endothelial corneal dystrophy by ROCK inhibitor (ripasudil). Am J Ophthalmol. 2021;224:185-99.

32. Kinoshita S, Koizumi N, Ueno M, et al. Injection of cultured cells with a ROCK inhibitor for bullous keratopathy. N Engl J Med. 2018;378:995-1003.

33. Tanihara $H$, Inoue $\mathrm{T}$, Yamamoto $\mathrm{T}$, et al. Additive intraocular pressure-lowering effects of the Rho kinase inhibitor ripasudil (K-115) combined with timolol or latanoprost: a report of 2 randomized clinical trials. JAMA Ophthalmol. 2015;133:755-61.

34. Futakuchi A, Morimoto T, Ikeda Y, et al. Intraocular pressure-lowering effects of ripasudil in uveitic glaucoma, exfoliation glaucoma, and steroid-induced glaucoma patients: ROCK-S, a multicentre historical cohort study. Sci Rep. 2020;10:10308.

35. Kusuhara S, Katsuyama A, Matsumiya W, et al. Efficacy and safety of ripasudil, a Rho-associated kinase inhibitor, in eyes with uveitic glaucoma. Graefes Arch Clin Exp Ophthalmol. 2018;256: 809-14.

36. Matsumura R, Inoue T, Matsumura A, et al. Efficacy of ripasudil as a second-line medication in addition to a prostaglandin analog in patients with exfoliation glaucoma: a pilot study. Clin Drug Investig. 2017;37:535-9.

37. Havens SJ, Gulati V. Neovascular glaucoma. Dev Ophthalmol. 2016;55:196-204. 